\title{
Shortened persistent homology for a biomedical retrieval system with relevance feedback *
}

\author{
Alessia Angeli ${ }^{1}$, Massimo Ferri ${ }^{2}$, Eleonora Monti ${ }^{1}$, and Ivan Tomba ${ }^{3}$ \\ 1 Dept. of Mathematics, Univ. Bologna, Italy \\ \{alessia.angeli, eleonora.monti5\}@studio.unibo.it \\ 2 Dept. of Mathematics and ARCES, Univ. Bologna, Italy massimo.ferri@unibo.it \\ 3 2R\&D Dept., CA-MI S.r.l., Via Ugo La Malfa 13, Pilastro di Langhirano PR, Italy \\ tomba.ivan@gmail.com
}

\begin{abstract}
This is the report of a preliminary study, in which a new coding of persistence diagrams and two relevance feedback methods, designed for use with persistent homology, are combined.
\end{abstract}

Keywords: Persistence diagram - Elementary symmetric function · Projected gradient.

\section{Introduction}

The interaction between a medical doctor and a smart machine must respect at least two requirements: Fast action and good integration with the human operator. In fact, the number of (hidden or evident, formal or intuitive) parameters in a diagnostic task is so high, that a good synthesis in short times is, at least for the moment, the field of a human expert; the machine can anyway offer a reliable, stable, powerful assistance.

This is the case of a system currently developed by Ca-Mi srl, an Italian company producing biomedical devices, in collaboration with the Universities of Bologna and Parma and with the Romagna Institute for Study and Cure of Tumors (IRST). The machine acquires the image of a dermatological lesion and retrieves a set of most similar images out of a database with sure diagnoses. The similarity is granted by a relatively recent geometric-topological technique: Persistent homology.

While the first experiments are very promising [7], there is still a wide gap between what the system and the doctor see as "similar". Therefore we and other researchers are studying a relevance feedback method for drawing the machine's formalization of similarity near the doctor's skilled view.

In this paper we present a preliminary study on a small public database $\left(\mathrm{PH}^{2}\right)$ of nevi and melanomas; our goal is to combine two relevance feedback methods expressly designed for persistent homology, with a new coding of one of its main tools, persistence diagrams.

\footnotetext{
* Article written within the activity of INdAM-GNSAGA.
} 
Content Based Image retrieval (CBIR) is a ripe and challenging research area [8]. Apart from annotation-based systems, much of the success of CBIR is tied with the use of histograms (of colours, directions, etc.). Topological descriptors have entered the game but are not yet fully employed [13]. On the other hand, we are aware of the incredible flourishing of Deep Learning in the areas of image understanding and of medical diagnosis; our interest in the techniques proposed here depends on the needs to work with a rather limited database, and to be able to control step-by-step how the system adapts to the user. Still we plan, as a future step, to combine persistent homology with Deep Learning - as several researchers already do - by feeding a neural net with persistence diagrams.

\section{Persistent homology}

Persistent homology is a branch of computational topology, of remarkable success in shape analysis and pattern recognition, above all for data of natural origin [5]. Its key idea is to analyze data through filtering functions, i.e. continuous functions $f$ defined on a suitable topological space $X$ with values e.g. in $\mathbb{R}$ (but sometimes in $\mathbb{R}^{n}$ or in a circle). Given a pair $(X, f)$, with $f: X \rightarrow \mathbb{R}$ continuous, for each $u \in \mathbb{R}$ the sublevel set $X_{u}$ is the set of elements of $X$ whose value through $f$ is less than or equal to $u$.

For each $X_{u}$ one can compute its homology modules $H_{r}\left(X_{u}\right)$, vector spaces which summarize the presence of "voids" of the different dimensions $r$ (connected components in the case $r=0$ ) and their relations.

Of course, if $u<v$ then $X_{u} \subseteq X_{v}$. There corresponds a linear map $\iota_{(u, v)}^{r}$ : $H_{r}\left(X_{u}\right) \rightarrow H_{r}\left(X_{v}\right)$. On $\Delta^{+}=\left\{(u, v) \in \mathbb{R}^{2} \mid u<v\right\}$ we can then define the $r$-Persistent Betti Number ( $r$-PBN) function

$$
\begin{aligned}
\beta_{(X, f)}^{r}: \Delta^{+} & \rightarrow \mathbb{Z} \\
(u, v) & \mapsto \operatorname{dim} \operatorname{Im}\left(\iota_{(u, v)}^{r}\right)
\end{aligned}
$$

All information carried by $r$-PBN's is condensed in some points (dubbed proper cornerpoints) and some half-lines (cornerlines); cornerlines are actually thought of as cornerpoints at infinity. Cornerpoints (proper and at infinity) build what is called the persistence diagram relative to dimension $r$. Figure 1 shows a letter " $\mathrm{M}$ " as space $X$, ordinate as function $f$ on the left, its 0-PBN function at the center and the corresponding persistence diagram on the right.

Remark 1. The theory also contemplates a multiplicity for cornerpoints (proper and at infinity); multiplicity higher than one is generally due to symmetries. We don't care about it in the present research, since all cornerpoints in our experiments have multiplicity one, as usual in diagrams coming from natural images.

Classification and retrieval of persistence diagrams (and consequently of the object they represent) is usually performed by the following distance, where persistence diagrams are completed by all points on the "diagonal" $\Delta=\{(u, v) \in$ $\mathbb{R} \mid u=v\}$. 

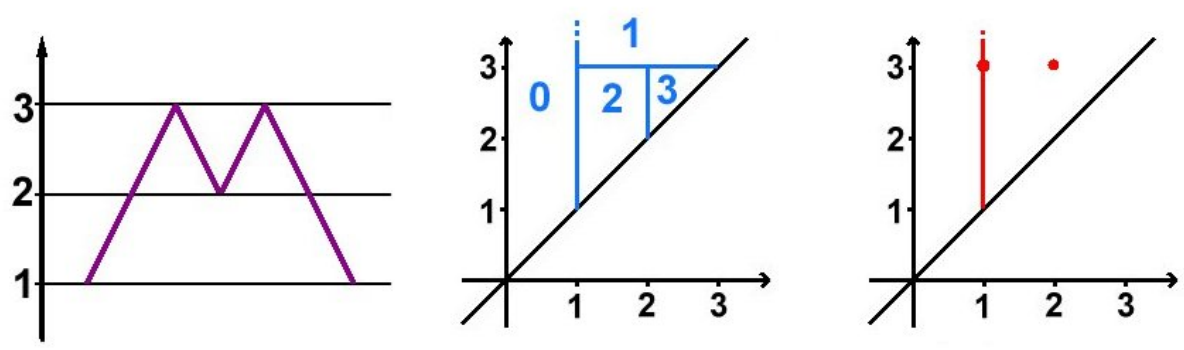

Fig. 1: Letter M, its 0-PBM function and the corresponding persistence diagram, relative to filtering function ordinate.

Definition 1. Bottleneck (or matching) distance.

Let $\mathcal{D}_{k}$ and $\mathcal{D}^{\prime}{ }_{k}$ be two persistence diagrams with a finite number of cornerpoints, the bottleneck distance $d_{B}\left(\mathcal{D}_{k}, \mathcal{D}^{\prime}{ }_{k}\right)$ is defined as

$$
d_{B}\left(\mathcal{D}_{k}, \mathcal{D}^{\prime}{ }_{k}\right)=\min _{\sigma} \max _{P \in \mathcal{D}_{k}} \hat{d}(P, \sigma(P))
$$

where $\sigma$ varies among all the bijections between $\mathcal{D}_{k}$ and $\mathcal{D}^{\prime}{ }_{k}$ and

$$
\hat{d}\left((u, v),\left(u^{\prime}, v^{\prime}\right)\right)=\min \left\{\max \left\{\left|u-u^{\prime}\right|,\left|v-v^{\prime}\right|\right\}, \max \left\{\frac{v-u}{2}, \frac{v^{\prime}-u^{\prime}}{2}\right\}\right\}
$$

given $(u, v) \in \mathcal{D}_{k}$ and $\left(u^{\prime}, v^{\prime}\right) \in \mathcal{D}^{\prime}{ }_{k}$.

For homology theory one can consult any text on algebraic topology, e.g. [10]. For persistent homology, two good references are [3, 4].

\section{Symmetric functions of warped persistence diagrams}

There are two main difficulties in comparing persistence diagrams. One is the fact that the diagonal $\Delta$ has a special role: Cornerpoints close to $\Delta$ ought to be considered close to each other. A second difficulty consists in the coding of a set of points in the plane: E.g. an intuitive coding like making a $2 M$ vector out of a set of $M$ points is highly unstable and is of no use in computing useful distances between persistence diagrams. In [1] we have tried to overcome both difficulties.

Following $[6,2]$, we have faced the first problem by two different transformations $T$ and $R$ which "warp" the plane, so that all $\Delta$ is sent to $(0,0)$, seen here as the complex number zero:

$$
T: \bar{\Delta}^{+} \rightarrow \mathbb{C}, \quad T(u, v)=\frac{v-u}{2}(\cos (\alpha)-\sin (\alpha)+i(\cos (\alpha)+\sin (\alpha)))
$$

where $\alpha=\sqrt{u^{2}+v^{2}}$. 


$$
R: \bar{\Delta}^{+} \rightarrow \mathbb{C}, \quad R(u, v)=\frac{v-u}{\sqrt{2}}(\cos (\theta)+i \sin (\theta))
$$

where $\theta=\pi(u+v)$.

Both $T$ and $R$ are continuous maps. See Figure 2 for a persistent diagram and its two images through $T$ and $R$.
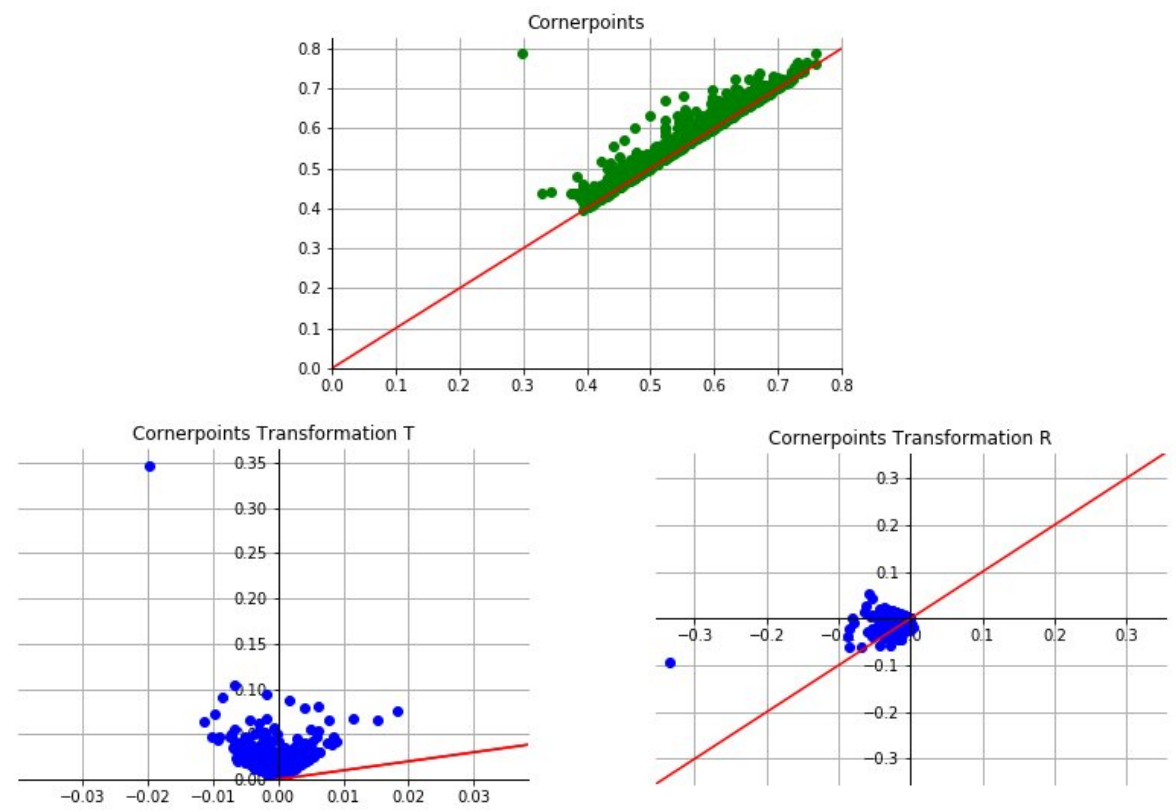

Fig. 2: A persistence diagram (above) and its images through $T$ (left) and $R$.

As for the second problem, following an idea of Claudia Landi $[6,2]$ we decided to encode each (transformed) persistence diagram $\mathcal{D}$ as the polynomial having the complex numbers, images of the cornerpoints, as roots. Actually, we form the vector having as components the elementary symmetric functions of those numbers (which, through Viète's formulas, equal the coefficients of that polynomial up to the sign) [11, Sect. IV.8]. So, the first component of the vector is the sum of all those numbers, the second one is the sum of all pairwise products, and so on. In order to take also cornerpoints at infinity into account, we have performed the following substitution. Given a cornerpoint at infinity (i.e. a cornerline) with abscissa $w$ of a persistence diagram $\mathcal{D}$, we substitute it with the point

$$
(w, \max \{v \mid(u, v) \text { is a cornerpoint of } \mathcal{D}\})
$$

Remark 2. Since cornerpoints near $\Delta$ generally represent noise, the two transformations were designed to wrap them around zero, so that the symmetric functions be scarcely affected by them. 
The elementary symmetric functions of large sets of complex numbers may show difference of up to six orders of magnitude. This might consistently alter the comparisons, so we actually formed, for each persistence diagram $\mathcal{D}$, the complex vector $a_{\mathcal{D}}$ whose $i$-th component $a_{\mathcal{D}}(i)$ is the $i$-th root of the $i$-th elementary symmetric function of the transformed cornerpoints, divided by the number of cornerpoints.

The advantage of using the vectors $a_{\mathcal{D}}$ instead of the original diagrams is that one can directly design a distance between complex vectors component-bycomponent (e.g. the $L^{1}$ distance we used), instead of considering all bijections between cornerpoint sets. Still, the computation of all the elementary symmetric functions would be too time consuming, so we performed in [1] some experiments by reducing the computation to the first $k$ components, $k \in\{5,10,20,50\}$, a small number compared with the hundreds of cornerpoints commonly found in the persistence diagrams of the examined images. Classification and retrieval of dermatological images using such "shortened" vectors was quite satisfactory with a dramatic time reduction.

\section{Modifying distances}

One of the major advantages of persistent homology is its modularity: By changing filtering function we change point of view on the shape of the objects under study and on the comparison criteria. Therefore the same data can be transformed into several pairs $(X, f)$, and for each we obtain distances. Both for classification and for retrieval, we need a single distance, so we have to blend the distances we have into one. There are two rather natural choices for that: either the maximum or the arithmetic average.

Maximum is the initial choice of [9]. An ongoing research, by some of the authors of the present paper, prefers the average instead. In fact, as hinted in the Introduction, the research is aimed at enhancing a device of acquisition and retrieval of dermatological images. The concept of "similarity" is (and has to remain!) highly subjective in the medical domain: We want to adapt the system to the physician, not the other way around. This can be done by modifying the weights of the different distances when building a single distance, to approximate the (pseudo)distance $\delta$ representing the dissimilarity as perceived by the user.

Our setting is: We have a set $X=\left\{x_{1}, \ldots, x_{N}\right\}$ of objects (in our case dermatological images) and $J$ descriptors $d^{(1)}, \ldots, d^{(J)}$ which give rise to an initial distance $D^{I N}$ between the objects in $X$. In our study, $D^{I N}$ is one of these two:

$$
D^{M A X}=\max \left\{d^{(1)}, \ldots, d^{(J)}\right\}, \quad D^{A V G}=\frac{d^{(1)}+\cdots+d^{(J)}}{J}
$$

Given a query $q$, the system retrieves the $L$ objects closest to $q$ with respect to $D^{I N}$, i.e. an $L$-tuple $X_{q}=\left(x_{i_{1}}, \ldots, x_{i_{L}}\right)$ such that $D^{I N}\left(q, x_{i_{1}}\right) \leq$ $\cdots \leq D^{I N}\left(q, x_{i_{L}}\right)$. The user is shown these objects and expresses his/her relevance feedback by giving a synthetic indication of the perceived dissimilarities $\delta\left(q, x_{i_{1}}\right), \ldots, \delta\left(q, x_{i_{L}}\right)$. 
While the Multilevel Relevance Feedback (MLRF) method proposed in [9] starts from $D^{M A X}$, then rescales the distances $d^{(i)}$ and takes the maximum, our Least Squares Relevance Feedback (LSRF) scheme start from $D^{A V G}$ and computes a new distance $D^{O U T}$ as

$$
D^{\text {OUT }}=\sum_{j=1}^{J} \lambda_{j} d^{(j)}, \quad \lambda_{j} \geq 0
$$

by minimizing the objective function

$$
g(\lambda)=\|\mathbf{d} \lambda-\delta\|_{2}^{2}
$$

where the $t$-th row of matrix $\mathbf{d}$ is formed by the distances $d^{(j)}\left(q, x_{i_{t}}\right), \lambda$ is the column matrix of the $\lambda_{j}$ and $\delta$ is the column matrix formed by $\delta\left(q, x_{i_{t}}\right)$ for $t=1, \ldots, L$ and $j=1, \ldots, J$.

Since this minimization problem might have multiple solutions, the vector of weights $\lambda_{j}$ in $D^{O U T}$ is obtained by iterating the Projected Gradient method. $D^{O U T}$ is the best possible distance approximating the users similarity distance $\delta$ from the given data in a least-square sense. More details on this procedure will be given in an article to come.

\section{Experimental results}

As a preliminary study, we have tried to combine the modularity of persistent homology with the fast computation of the short vectors of symmetric functions of the transformed cornerpoints, with the adapting weights of relevance feedback.

We experimented with a small public database, $P H^{2}$ [12], containing 8-bit RGB, $768 \times 560$ pixels images of 80 common nevi, 80 atypical nevi, and 40 melanomas. We used 19 distances: 8 coming from simple morphological parameters, 11 coming from as many filtering functions; see Table 1 for a description of these features $[7,1]$. For each image, we built the 11 persistence diagrams, performed one of the transformations $T$ and $R$ (see Sect. 3), and computed the 11 corresponding vectors, limited to length $k=20$.

In normal operation, the user will give his/her feedback as follows. For $j=$ $1, \ldots, L$, the user is asked to assign a similarity judgement (where 0 stands for "dissimilar" and 1 for "similar") to the pair $\left(q, x_{i_{j}}\right)$ with respect to three different aspects of the skin lesion (boundary/shape, colours, texture). This results in a similarity vote $v$ in the range $\{0,1,2,3\}$ which is transformed into $\delta$ by the following formula:

$$
\delta\left(q, x_{i_{j}}\right)=\max \left\{0, \quad D^{I N}\left(q, x_{i_{1}}\right)+\frac{4(3-v)-1}{10}\left(D^{I N}\left(q, x_{i_{1}}\right)-D^{I N}\left(q, x_{i_{L}}\right)\right)\right\}
$$

We finally retrieve again the $L$ closest objects according to the modified distance (see Section 4). 


\begin{tabular}{cc}
\hline persistence features & morphological features \\
\hline Light Intensity & Colour Histogram \\
Blue & Form Factor \\
Green & Haralick's Circularity \\
Red & Asymmetry \\
Excess Blue & Ellipticity \\
Excess Green & Eccentricity \\
Excess Red & Diameter \\
Light Intensity 2 & Colour Entropy \\
Boundary Light Intensity & \\
Boundary & \\
Boundary 2 & \\
\hline
\end{tabular}

Table 1: Features.

In the present research $L=10$ and the retrieval is performed by the "leave one out" scheme. Not having a real relevance feedback by a physician, we assign a retrieved image the maximal similarity vote $v=3$ if it shares the same histological diagnosis of the query, and the minimal similarity vote $v=0$ otherwise.

Assessment of the retrieval is performed by counting how many, of the retrieved lesions, have the same diagnosis of the query before and after the feedback. This is summarized in Table 2 by summing these scores for all images of the database as queries, starting from $D^{M A X}$ and then applying the MLRF scheme, starting from $D^{A V G}$ and applying our LSRF method.

\begin{tabular}{|l||c|c|c||c|c|c|}
\hline & with $D^{M A X}$ & MLRF & difference & with $D^{A V G}$ & LSRF & difference \\
\hline Transf. $T$ & 1694 & 1776 & 82 & 1729 & 1856 & 127 \\
Transf. $R$ & 1869 & 1762 & 73 & 1735 & 1857 & 122 \\
\hline
\end{tabular}

Table 2: Sums of scores with the two considered methods of relevance feedback.

As we can see, the two warping transformations yield similar results. $D^{A V G}$ performs better than $D^{M A X}$ and LSRF produces a better improvement than MLRF.

\section{Conclusions}

We have compared - on a small public database of nevi and melanomas - two relevance feedback methods, conceived for a retrieval system based on persistent homology, combined with a computation reduction based on elementary symmetric functions of warped persistence diagrams. A weighted average of distances, with weights obtained by a standard optimization method, seems to 
perform better. The experiment will be extended to larger databases and with real feedback from expert dermatologists.

\section{References}

1. A. Angeli, M. Ferri, and I. Tomba. Symmetric functions for fast image retrieval with persistent homology. preprint, 2018.

2. B. Di Fabio and M. Ferri. Comparing persistence diagrams through complex vectors. In International Conference on Image Analysis and Processing, pages 294305. Springer, 2015.

3. H. Edelsbrunner and J. Harer. Persistent homology - a survey. In Surveys on discrete and computational geometry, volume 453 of Contemp. Math., pages 257282. Amer. Math. Soc., Providence, RI, 2008.

4. H. Edelsbrunner and J. Harer. Computational Topology: An Introduction. American Mathematical Society, 2009.

5. M. Ferri. Persistent Topology for Natural Data Analysis - A Survey, pages 117133. Springer International Publishing, Cham, 2017.

6. M. Ferri and C. Landi. Representing size functions by complex polynomials. In Proc. Math. Met. in Pattern Recognition, volume 9, pages 16-19, 1999.

7. M. Ferri, I. Tomba, A. Visotti, and I. Stanganelli. A feasibility study for a persistent homology-based k-nearest neighbor search algorithm in melanoma detection. Journal of Mathematical Imaging and Vision, 57(3):324-339, 2017.

8. N. Ghosh, S. Agrawal, and M. Motwani. A survey of feature extraction for contentbased image retrieval system. In Proceedings of International Conference on Recent Advancement on Computer and Communication, pages 305-313. Springer, 2018.

9. D. Giorgi, P. Frosini, M. Spagnuolo, and B. Falcidieno. 3D relevance feedback via multilevel relevance judgements. The Visual Computer, 26(10):1321-1338, 2010.

10. A. Hatcher. Algebraic topology. 2002, volume 606. 2002.

11. S. Lang. Undergraduate algebra. Springer Science \& Business Media, 2005.

12. T. Mendonça, P. M. Ferreira, J. S. Marques, A. R. Marcal, and J. Rozeira. Ph 2-a dermoscopic image database for research and benchmarking. In Engineering in Medicine and Biology Society (EMBC), 2013 35th Annual International Conference of the IEEE, pages 5437-5440. IEEE, 2013.

13. M. Zeppelzauer, B. Zieliński, M. Juda, and M. Seidl. A study on topological descriptors for the analysis of $3 \mathrm{~d}$ surface texture. Computer Vision and Image Understanding, 2017. 